
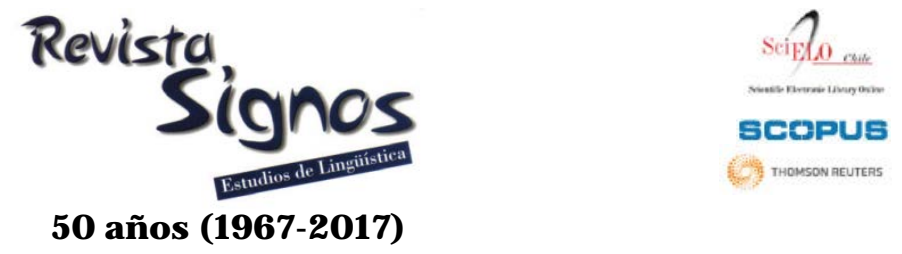

50 años $(1967-2017)$

\title{
¿Se escribe junto o separado? Evolución en el uso de los espacios en blanco en textos de niños que inician su alfabetización ${ }^{1}$
}

\author{
Together or separate? The development of the use of spaces \\ between words in pupil's texts
}

\author{
Marta Alegría Ugarte \\ Universidad Alberto Hurtado \\ CHILE \\ malegria@uahurtado.cl
}

Celia Díaz Argüero
Universidad Autónoma DE MÉxico
MÉxico
calyad@unam.mx

Recibido: 26/IV/2015 / Aceptado: 08/VII/2016

\section{Resumen}

Diversos trabajos, realizados desde una perspectiva psicogenética y psicolingüística, han permitido conocer algunas características de la segmentación entre palabras que hacen los niños al inicio de la alfabetización. En este trabajo se presenta un análisis cuantitativo y cualitativo de 90 textos de niños mexicanos, de segundo y cuarto grados de educación primaria. El propósito del análisis es aportar elementos para identificar la existencia de un posible patrón evolutivo en el aprendizaje de la segmentación entre palabras de la escritura del español. El trabajo se centra en el análisis de las segmentaciones no convencionales (SNC) que hacen los niños y en la identificación de los posibles criterios que guían esas segmentaciones. Los hallazgos confirman la hipótesis inicial: las segmentaciones no convencionales son el resultado de un ejercicio racional que realizan los niños en su esfuerzo por comprender la naturaleza de las unidades que se separan en la escritura y no son 'errores ortográficos'. En términos evolutivos, los resultados permiten ver que, en niveles iniciales, los niños exploran diversos criterios para separar las palabras, y que, a medida aumenta su conocimiento de la escritura, irán abandonando algunos de esos criterios.

Palabras Clave: Alfabetización inicial, segmentación en la escritura, palabras léxicas y funcionales, ortografía. 


\begin{abstract}
Several studies, conducted within a psycholinguistic and psychogenetic theoretical framework, have made it known some of the characteristics related to segmentation between words that children write when in their literacy first stages. This work presents, both, a quantitative and a qualitative analysis of 90 texts written by Mexican children who are in second and fourth grade in elementary school. The purpose of this study is to provide some ideas in order to identify the existence of a possible evolutionary pattern in learning segmentation between words in Spanish writing. This work focuses on the analysis of unconventional word segmentation (SNC) that children make and on the identification of possible criteria that guide these segmentations. The findings confirm the initial hypothesis: unconventional word segmentation observed in children's writing is the result of a rational exercise carried out by children in their effort to understand the nature of the graphic units instead of 'spelling errors'. In evolutionary terms, the results show that children explore, in initial levels, different criteria to separate words, and, as their writing knowledge increases, some of these criteria will be discarded.
\end{abstract}

Key Words: Initial literacy, text segmentation, lexical and functional words, orthography.

\title{
INTRODUCCIÓN
}

La ortografía es un asunto importante en diversos ámbitos de la sociedad. En la vida académica y laboral, las 'faltas de ortografía' pueden llegar a ser causa de desacreditación y desprestigio profesional. En el campo educativo, es tema de preocupación de diversos actores: desde los profesores responsables de enseñar y evaluar contenidos ortográficos hasta investigadores que buscan comprender los procesos de adquisición y las condiciones didácticas que favorecen su aprendizaje. Diversos estudios muestran que es un contenido complejo de dominar por los niños (OREALC/UNESCO, 2010; Sotomayor, Molina, Bedwell \& Hernández, 2013).

Los estudios sobre segmentación en palabras en la escritura infantil, y en ortografía en general, han sido tradicionalmente abordados desde una perspectiva normativa. Sin embrago, los trabajos de Ferreiro y Teberosky (1979) marcaron el inicio de una serie de estudios desarrollados desde una perspectiva psicogenética y psicolingüística, que se preocupa por analizar los 'desaciertos' en la escritura de los niños no desde la mirada del adulto alfabetizado sino desde una perspectiva comprensiva, que busca develar las razones que guían las decisiones de los sujetos y las ideas que construyen para comprender la naturaleza y el funcionamiento del lenguaje escrito.

En esta perspectiva, las investigaciones que se han ocupado de estudiar la evolución del conocimiento infantil de la segmentación han evidenciado que los niños utilizan un conocimiento metalingüístico implícito para reflexionar sobre la naturaleza de los elementos que forman las palabras y resolver los problemas que le plantea la segmentación de los textos (Díaz, 1992; Ferreiro \& Pontecorvo, 1996; Báez, 1999; Zamudio, 2004; Vaca, 2005; Bigas, 2006; Alegría, 2014). Las conclusiones de estos 
estudios han identificado algunos rasgos significativos de la posible trayectoria evolutiva que siguen los niños hasta lograr una segmentación convencional. En continuidad con estas investigaciones, el estudio que aquí se presenta tiene como propósito aportar elementos para identificar la existencia de un posible patrón evolutivo en el aprendizaje de la segmentación entre palabras de la escritura del español. El trabajo se centra en el análisis de las segmentaciones no convencionales (SNC) que hacen los niños y en la identificación de posibles criterios que guían esas segmentaciones. Para hacerlo, nos hemos planteado las siguientes preguntas: ¿qué tipos de segmentaciones no convencionales (SNC) hacen los niños que muestran diferentes dominios de la segmentación convencional de palabras?, ¿qué tipo de palabras se ven involucradas en estos fenómenos? y ¿qué criterios podrían estar orientando las decisiones de los niños?

Avanzar en la comprensión del aprendizaje infantil de la segmentación tiene importancia teórica y alcances para la didáctica. Los niños tendrán más posibilidad de avanzar en el aprendizaje si el docente logra plantearles situaciones problemáticas que los alumnos puedan comprender y a la vez les resulten desafiantes y les exijan la construcción de nuevos conocimientos. Esto solo será posible si contamos con más investigaciones que se propongan no solo tipificar y cuantificar errores ortográficos, sino que contribuyan a la comprensión de las razones que podrían estar detrás de las decisiones que toman los niños cuando escriben.

\section{Marco teórico}

\subsection{La segmentación en palabras en la historia de la escritura alfabética}

La historia de la escritura alfabética ofrece interesantes elementos que permiten reflexionar sobre el proceso que enfrentan los sujetos en alfabetización. Aunque hoy reconocemos la segmentación como problema de la escritura, su origen estuvo más bien asociado a la lectura. Hasta la Edad Media el latín se escribía sin espacios en blanco entre palabras, frases o párrafos y sin signos de puntuación. En esta época, la propagación del cristianismo constituyó un evento decisivo en el surgimiento de diversas marcas presentes en nuestra escritura, como son los espacios en blanco y el punto. En el siglo VIII d.C., monjes irlandeses y anglosajones, para quienes el latín constituía una lengua extranjera, se vieron en la necesidad de introducir marcas que facilitaran la interpretación y copia de los manuscritos griegos. Saenger (1997), Pérez (2006) y Zamudio (2010) señalan que se trataba de lectores para quienes el latín les resultaba una expresión más visible que audible, que la página se les presentaba con un orden propio, independiente y diferente del lenguaje oral y que introdujeron los espacios en blanco con el propósito de identificar unidades de sentido y facilitar la comprensión de los textos. 
De modo similar a lo que se observa en los escritos infantiles, la oscilación en los criterios de segmentación estuvo presente en la historia de la escritura. En el largo periodo de transición de la scriptura continua a la escritura con separaciones de palabras se emplearon diversos modos de agrupamientos de letras, como son los agrupamientos azarosos de letras y sílabas y agrupamientos jerárquicos de letras y de palabras. Asimismo, la difusión y consolidación de la segmentación convencional de las palabras fue un proceso lento y gradual. La separación de las palabras llegó primero a los territorios del norte de Francia, los Países Bajos y Normandía, es decir aquellas regiones que tenían mayor intercambio con los monasterios insulares, llegando a Italia a finales del siglo IX y sistematizándose su uso en el siglo X d. C.

\subsection{La palabra en la Lingüística}

La 'palabra' ha sido tema permanente de debate para la Lingǘstica, ciencia que más bien ha buscado hace tiempo sustituirla con términos especializados y se ha señalado que el morfema es la unidad mínima de la lengua (Ferreiro, 2012). Sin embargo, descartarla como unidad teórica no ha resuelto los problemas que implica su definición. Según señalan algunos lingüistas (Fruyt \& Reichler-Béguelin, 1990; Blanche-Benveniste, 1998; Béguelin, 2002; Zamudio, 2010) la diversidad de criterios que se han propuesto son tan independientes y diferentes unos de otros que no confluyen en una misma unidad y no coinciden con lo que los hablantes definen como palabra.

La palabra como unidad gráfica es, según Fruyt y Reichler-Béguelin (1990), la definición adoptada como la más satisfactoria para la corriente francesa. Desde esta perspectiva, la definición implica remitirse a la escritura sin hacer referencia a la lengua oral. Propone que palabra es serie de letras separadas por espacios en blanco. Al respecto, Ferreiro y Pontecorvo (1996) señalan que las investigaciones en lectura han mostrado que los espacios en blanco permiten al lector identificar unidades significativas y unidades que, que sin tener significado autónomo, afectan el significado de los grupos nominales y verbales. Aunque estas autoras reconocen que la escritura ofrece la mejor definición práctica (no teórica) de palabra, señalan que existen zonas de incertidumbre en la definición dentro de una misma lengua, por ejemplo, cuando una misma idea conceptual se representa usando dos espacios (antes de ayer) o ningún espacio (anteayer). Asimismo, los pronombres clíticos dan cuenta de una asistematicidad del español, en los que la escritura de estas partículas, unidas o separadas del verbo, depende de la posición que ocupen: enclítica (dámelo) o proclítica (me lo da) y no está determinada por una cuestión semántica ni gramatical. 


\subsection{Noción de palabra y segmentaciones en la escritura infantil: Estudios realizados desde una perspectiva psicolingüística}

Qué tipos de segmentaciones realizan los niños y cómo construyen la noción de 'palabra' han sido preocupaciones centrales de algunas investigaciones psicolingüísticas desarrolladas en las últimas dos décadas. Respecto a la noción de palabra, estudios realizados con niños prealfabéticos de 4 y 5 años señalan que 'nombre' y 'se llama' son términos de uso espontáneo y de fácil comprensión, en tanto que el término 'palabra' es ajeno a la terminología infantil, difícil de usar y más complejo aún de definir. En este sentido, difícilmente puede hablarse de una 'noción prealfabética de palabra', más bien 'palabra' evocaría a una diversidad de unidades de distinta naturaleza y principalmente haría referencias a los 'nombres' (Ferreiro \& Vernon, 1992).

Trabajos centrados en el análisis de la naturaleza de las segmentaciones, señalan que, con independencia de la lengua y nivel educativo, los niños producen escritos con 'hiposegmentaciones', 'hipersegmentaciones', así como la combinación de ambas. Las 'hiposegmentaciones' se refieren a escrituras con un menor número de segmentaciones de las que marca la norma escrita, de manera que se encuentran una o más palabras gráficas unidas; mientras que las 'hipersegmentaciones' permiten denominar escrituras con un número mayor de segmentaciones de las convencionales $y$, en consecuencia dan lugar a palabras gráficas fragmentadas. Desde una perspectiva cuantitativa, el fenómeno de la 'hiposegmentación' es el que se presenta con mayor frecuencia, mientras que las 'hipersegmentaciones' tienden a ser menos habituales. Por su parte, análisis de corte cualitativo indican que la mayor parte de las 'hiposegmentaciones' se producen frente a palabras de pocas letras (una, dos o tres) y que involucran predominantemente palabras gramaticales que carecen de significado pleno: preposiciones, conjunciones, artículos y pronombres clíticos (Ferreiro \& Teberosky, 1979; Díaz, 1992; Ferreiro \& Pontecorvo, 1996; Báez, 1999; Zamudio, 2004; Vaca, 2005; Bigas, 2006; González, 2009; Sotomayor et al., 2013; Reyes, 2014).

Otros estudios tratan de mostrar los criterios que pueden estar detrás de las segmentaciones que producen los niños. Zamudio (2004) y Querejeta, Piacente, Guerrero y Alva (2013) atribuyen al uso de criterios o indicios fonológicos, gráficos y de significación la mayor parte de las segmentaciones no convencionales infantiles. Por su parte, Báez (1999), que trabaja con niños con comprensión incipiente del sistema de escritura y con niños con mayor dominio de los aspectos ortográficos, afirma que los primeros tienden a fundamentar sus recortes en criterios gráficos, mientras que los niños con mayor conocimiento de la escritura lo hacen con argumentos de tipo sintáctico y semántico. Esta perspectiva psicolingüística es el marco teórico dentro del cual se realiza la presente investigación. 


\section{Marco metodológico}

\subsection{Casos e instrumento}

Analizamos 90 textos escritos por alumnos que iniciaban segundo (entre 7 y 8 años) y cuarto grado (entre 9 y 10 años) de educación primaria, en escuelas públicas ubicadas en zonas urbanas de Nayarit, México. Estos textos forman parte del CEELE (Corpus electrónico para el estudio del lenguaje escrito)2. Para obtenerlos, se leyó a los niños una historia que motivó su interés por describir su escuela y se les pidió que elaboraran un texto para que otros niños pudieran conocer las características de su entorno. En su escrito, debían responder a: ‘¿quién soy?’, ‘¿cómo es mi escuela?’, ‘¿cómo llegamos a la escuela?', ‘qué hacemos en la escuela?’ Los niños no tuvieron oportunidad de revisar su escrito ni de reescribirlo, de manera que se analizó la primera y única versión del texto.

\subsection{Descripción del proceso de análisis}

\subsubsection{Transliteración y etiquetado}

Para el procesamiento de los textos se realizó transliteración y etiquetado. La transliteración es una trascripción digital de las escrituras en formato Bloc de Notas, que conserva todas las decisiones gráficas de los niños durante la producción de sus textos: letras empleadas, espacios gráficos, signos de puntuación y cambios de renglón (Zamudio \& Díaz, 2011). El etiquetado en XML (eXtensible Markup Language 'lenguaje de marcas extensible') permite marcar los fenómenos identificados (acentuación, segmentación entre palabras, sustitución, agregado u omisión de grafías, cambios de mayúsculas/minúsculas, etc.) y elaborar una versión normalizada del texto. El corpus electrónico cuenta con una interfase que permite agrupar automáticamente las segmentaciones no convencionales (SNC) en tres grandes categorías identificadas en otras investigaciones: 'hiposegmentaciones' (uniones de secuencias gráficas que deberían estar separadas), 'hipersegmentaciones' (fragmentación de secuencias gráficas que deberían estar unidas) e 'hipo-hipersegmentaciones' (aparición de los dos fenómenos anteriores en dos palabras contiguas).

\subsubsection{Subcategorías de análisis}

Para analizar el trabajo de los niños fue necesario especificar los tipos de 'hiposegmentaciones' o ‘hipersegmentaciones' encontradas en los textos. Para ello se definieron subcategorías que están relacionadas con diferentes elementos. Por ejemplo: el tipo de estructura sintáctica o las categorías de palabras involucradas (funcionales/gramaticales o léxicas). Consideramos palabras léxicas a los sustantivos, adjetivos, verbos y adverbios. Y, palabras gramaticales o funcionales a los artículos, pronombres, conjunciones y preposiciones. En algunos caso fue necesario hacer referencia a la estructura morfológica de las palabras o a sus características gráficas. 
Identificamos 6 subcategorías de 'hiposegmentación' y 7 de 'hipersegmentaciones'. En las tablas 1 y 2 se presentan dichas subcategorías.

Tabla 1. Subcategorías de 'hiposegmentación'.

\begin{tabular}{|c|c|c|}
\hline Subcategoría & Elementos que la integran & Ejemplo \\
\hline HIPO PF+PL & $\begin{array}{c}\text { Palabra funcional + palabra } \\
\text { léxica }\end{array}$ & $\begin{array}{c}\text { y mi hermano seyama Oscar } \\
\text { (Y mi hermano se llama Oscar) }\end{array}$ \\
\hline HIPO PF+PF & $\begin{array}{c}\text { Palabra funcional + palabra } \\
\text { funcional }\end{array}$ & $\begin{array}{c}\text { ami me lleva mi Papá a la escuela } \\
\text { (A mí me lleva mi papá a la escuela) }\end{array}$ \\
\hline HIPO PL+PL & $\begin{array}{c}\text { Palabra léxica + palabra } \\
\text { léxica }\end{array}$ & $\begin{array}{c}\text { mi hermano se llama juanPablo } \\
\text { (Mi hermano se llama Juan Pablo) }\end{array}$ \\
\hline HIPO PL+PF & $\begin{array}{c}\text { Palabra léxica + palabra } \\
\text { funcional }\end{array}$ & $\begin{array}{c}\text { asemos educasion artisticay ase mos } \\
\text { matemáticas }\end{array}$ \\
\hline HIPO USC & $\begin{array}{c}\text { Dos o más palabras que } \\
\text { integran una unidad } \\
\text { sintáctica completa }\end{array}$ & $\begin{array}{c}\text { (hacemos educán artística y hacemos } \\
\text { matemáticas) }\end{array}$ \\
\hline HIPO Otros & $\begin{array}{c}\text { Dos o más palabras que no } \\
\text { corresponden a ninguna de } \\
\text { las subcategorías anteriores }\end{array}$ & $\begin{array}{c}\text { comatellamas } \\
\text { (¿Cómo te llamas?) }\end{array}$ \\
\hline
\end{tabular}

Tabla 2. Subcategorías de 'hipersegmentación'.

\begin{tabular}{|c|c|c|}
\hline Subcategoría & Elementos que la integran & $\begin{array}{l}\text { Ejemplo } \\
\end{array}$ \\
\hline HIPER PA & $\begin{array}{c}\text { Secuencias de letras que, en } \\
\text { otros contextos, podrían } \\
\text { funcionar como palabras } \\
\text { independientes }\end{array}$ & $\begin{array}{l}\text { aqui en Santa. Ma. del oro no a-se calor } \\
\text { pero en la noche a se mucho frio } \\
\text { (Aquí en Santa María del Oro no hace calor } \\
\text { pero en la noche hace mucho frío) }\end{array}$ \\
\hline $\begin{array}{l}\text { HIPER } \\
\text { C SIL }\end{array}$ & $\begin{array}{l}\text { Secuencias de letras que se } \\
\text { corresponden con cortes } \\
\text { silábicos }\end{array}$ & $\begin{array}{l}\text { Como arros y bri go Les } \\
\text { (como arroz y frijoles) }\end{array}$ \\
\hline HIPER DV & Desinencias verbales & $\begin{array}{c}\text { tene mos Libros de La biblioteca ase mos } \\
\text { trabajos } \\
\text { (Tenemos libros de la biblioteca, hacemos } \\
\text { trabajos) }\end{array}$ \\
\hline $\begin{array}{c}\text { HIPER } \\
\text { PLURAL } \\
\end{array}$ & Morfema de plural & $\begin{array}{l}\begin{array}{l}\text { salimo s } \\
\text { (salimos) }\end{array} \\
\end{array}$ \\
\hline HIPER AF & Afijo de una palabra & $\begin{array}{l}\text { re cortamos } \\
\text { (recortamos) }\end{array}$ \\
\hline $\begin{array}{l}\text { HIPER SUF } \\
\text {-MENTE }\end{array}$ & $\begin{array}{l}\text { Morfema 'mente' en } \\
\text { adverbios }\end{array}$ & $\begin{array}{l}\text { me gusta venir a la escuela diaria mente } \\
\text { (me gusta venir a la escuela diariamente) }\end{array}$ \\
\hline HIPER Otros & $\begin{array}{l}\text { Letras o secuencias gráficas } \\
\text { que no corresponden a } \\
\text { ninguna de las subcategorías } \\
\text { anteriores }\end{array}$ & $\begin{array}{c}\text { yo Naci en la Capital de tepic en el s eg uro } \\
\text { social } \\
\text { (Yo nací en la capital de Tepic en el seguro } \\
\text { social) }\end{array}$ \\
\hline
\end{tabular}




\subsubsection{Análisis}

En el centro de este estudio está la preocupación por el aspecto evolutivo así como por entender cuáles son los criterios que guían las decisiones de los niños al segmentar sus textos. Para analizar el trabajo de los niños con diferentes niveles de conocimiento, los agrupamos considerando dos criterios simultáneamente: la escolaridad (y por tanto también su edad) y el nivel de conocimiento sobre la segmentación. Para clasificarlos de acuerdo con este segundo criterio, ordenamos sus textos según la proporción de segmentaciones no convencionales (SNC) en relación al total de palabras que los integraban, desde los que mostraban más bajas proporciones de $\mathrm{SNC}(0,12 \%$ en segundo grado y $0 \%$ en cuarto grado), es decir aquellos que mostraban una segmentación muy cercana a la convencional, hasta a aquellos que tenían las más altas proporciones de SNC (18\% en segundo grado a $22 \%$ en cuarto). Después los agrupamos por deciles ${ }^{3}$. Esta distribución se puede observar en la Tabla 3.

Tabla 3. Porcentaje promedio de SNC en relación al total de palabras. Clasificación por grado escolar.

\begin{tabular}{|c|c|c|}
\hline \multirow{2}{*}{ Deciles } & \multicolumn{2}{|c|}{$\%$ promedio de SNC } \\
\cline { 2 - 3 } & Textos de segundo grado & Textos de cuarto grado \\
\hline $1^{\circ}$ Decil & $0,12 \%$ & $0,00 \%$ \\
\hline $2^{\circ}$ Decil & $1,10 \%$ & $0,68 \%$ \\
\hline $3^{\circ}$ Decil & $1,96 \%$ & $1,33 \%$ \\
\hline $4^{\circ}$ Decil & $2,94 \%$ & $1,73 \%$ \\
\hline $5^{\circ}$ Decil & $4,26 \%$ & $2,50 \%$ \\
\hline $6^{\circ}$ Decil & $5,56 \%$ & $4,18 \%$ \\
\hline $7^{\circ}$ Decil & $7,10 \%$ & $4,85 \%$ \\
\hline $8^{\circ}$ Decil & $10,20 \%$ & $7,33 \%$ \\
\hline $9^{\circ}$ Decil & $13,24 \%$ & $11,48 \%$ \\
\hline $10^{\circ}$ Decil & $18,00 \%$ & $22,48 \%$ \\
\hline & & \\
\hline
\end{tabular}

En búsqueda de una posible tendencia o patrón evolutivo, analizamos y comparamos la distribución de las categorías y subcategorías propuestas por grado y por decil. Realizamos fundamentalmente análisis de frecuencias.

\section{Resultados y discusión}

\subsection{Tipos de SNC}

Considerando el nivel de escolaridad como criterio de agrupación, encontramos similitudes importantes entre los textos de ambos grados. Éstas pueden observarse en la Tabla 4. 
Tabla 4. Tipos de SNC en textos según grado escolar.

\begin{tabular}{|c|c|c|}
\hline Grado & $2^{\circ}$ grado & $4^{\circ}$ grado \\
\hline Hipos de SNC & $61,2 \%$ & $64,0 \%$ \\
\hline Hipersegmentaciones & $34,2 \%$ & $32,3 \%$ \\
\hline Hipo-hipersegmentanes & $4,6 \%$ & $3,8 \%$ \\
\hline
\end{tabular}

Tal como lo constataran otros autores, la 'hiposegmentación' es el tipo más frecuente de SNC, seguido por la 'hipersegmentación' y, en proporciones mucho más bajas, se encuentran las 'hipo-hipersegmentaciones'. En el Gráfico 1 podremos observar, la distribución cada tipo de SNC por decil, en cada uno de los grados escolares.

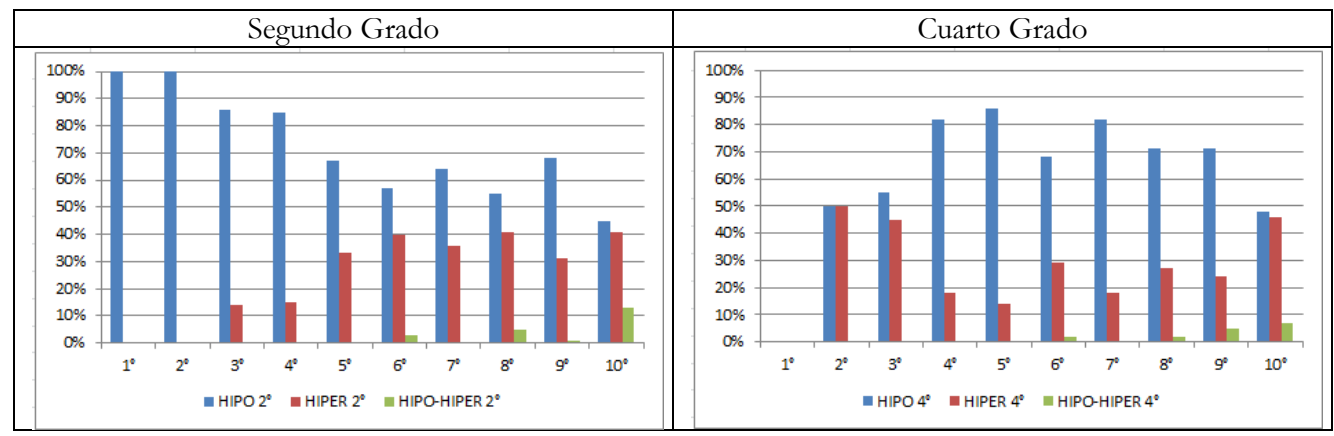

Gráfico 1. Tipos de SNC en segundo y cuarto grado. Distribución por decil.

Los datos que se observan en este gráfico son muy interesantes. Encontramos que, en todos los deciles, en ambos grados (menos en el $2^{\circ}$ decil de cuarto), la 'hiposegmentación' es el fenómeno más frecuente. Esta situación ha sido reportada por otras investigaciones con niños de diferentes lenguas (Ferreiro \& Pontecorvo, 1996), grados escolares (Báez, 1999) y también con adultos (Rayas, 2009), con tareas de escritura de textos y frases, de forma libre y al dictado. La organización de los datos en deciles permite observar que, en segundo grado, los textos que tienen en promedio proporciones más bajas de SNC (1 ${ }^{\circ}$ y $2^{\circ}$ decil) contienen exclusivamente 'hiposegmentaciones', la predominancia de las 'hiposegmentaciones' con respecto a las 'hipersegmentaciones' disminuye en los demás deciles e inclusive en el último $\left(10^{\circ}\right)$ es casi equivalente a las 'hiposegmentaciones'. En cuarto grado en cambio, no se observa un comportamiento regular de las 'hiposegmentaciones', de manera que en algunos casos se observan cantidades equivalentes de ambas categorías de $\mathrm{SNC}\left(2^{\circ}, 3^{\circ}\right.$ y $10^{\circ}$ decil), mientras que en los demás deciles encontramos una clara predominancia de las 'hiposegmentaciones' por sobre las 'hipersegmentaciones'.

Respecto de las 'hipersegmentaciones', se observa que en todos los deciles, en ambos grados (menos en el $1^{\circ}$ decil de cuarto), es el segundo fenómeno más frecuente. Esta tendencia coincide con lo encontrado en otros estudios (Ferreiro \& 
Pontecorvo, 1996; Báez, 1999; Rayas, 2009). En relación a este tipo de segmentación, una vez más el factor proporción de SNC nos permite constatar un comportamiento interesante. En segundo grado, los porcentajes más altos de 'hipersegmentaciones' se encuentran del $5^{\circ}$ al $10^{\circ}$ decil. Así, en segundo grado y de modo contrario a lo que se observa con la 'hiposegmentación', la 'hipersegmentación' es una respuesta que surge con más frecuencia en niños con un conocimiento más incipiente de la segmentación. En cuarto grado, en cambio, no se observa una tendencia clara, observamos deciles con porcentajes equivalentes de 'hiposegmentaciones' e 'hipersegmentación' $\left(2^{\circ}, 3^{\circ}\right.$ y $\left.10^{\circ}\right)$ y otros casos con porcentajes que no alcanzan el $20 \%\left(4^{\circ}, 6^{\circ}\right.$ y $8^{\circ}$ decil $)$.

Por último, la distribución de los porcentajes de 'hipo-hipersegmentaciones' resulta interesante. Se trata de un fenómeno poco frecuente, que se observa exclusivamente en los casos con menos conocimiento de la segmentación convencional, en ambos grados. Que estos tipos de cortes se concentren en los últimos deciles, y que además en algunos de estos subgrupos se observen proporciones semejantes de 'hiposegmentaciones' e 'hipersegmentaciones' (ver $6^{\circ}, 8^{\circ}$ y $10^{\circ}$ decil de segundo y $10^{\circ}$ decil de cuarto), da cuenta de que los niños aún no han identificado los límites plausibles en las secuencias gráficas. En relación a lo observado hasta ahora, sobre todo desde el $6^{\circ}$ al $10^{\circ}$ decil en ambos grados, compartimos lo señalado por Ferreiro y Pontecorvo (1996: 61):

"La inestabilidad segmental que se observa en los grupos con más altos porcentajes de segmentaciones no convencionales puede deberse a una ausencia de conocimiento o una comprensión de la producción escrita como espacio de prueba de segmentaciones posibles, según una multiplicidad de criterios aún no jerarquizados”.

Sin embargo, ¿serán similares las 'hiposegmentaciones' de los niños que realizan exclusivamente este tipo de SNC a las de aquellos que realizan 'hiposegmentaciones', además de 'hipersegmentaciones'?, ¿afectarán a los mismos tipos de palabras?, ¿estarán guiadas por criterios similares? Para dar respuesta a estas preguntas hicimos un análisis cualitativo de los datos, tomando como parámetro las subcategorías presentadas en las tablas 1 y 2 .

\subsection{Resultados por subcategorías}

Por razones de espacio y debido a que no encontramos grandes diferencias entre segundo y cuarto, en las siguientes tablas mostraremos los datos de ambos grados de manera conjunta, aunque en los comentarios explicativos haremos referencia a lo que ocurrió en cada uno de los grados ${ }^{4}$. En los siguientes cuadros veremos cómo se distribuyen las SNC. En la Tabla 5 presentaremos las 'hiposegmentaciones' y posteriormente, en la Tabla 6, las 'hipersegmentaciones'. Con la finalidad de facilitar la comparación, presentamos los datos expresados tanto en frecuencias como en porcentajes. 


\subsubsection{Hiposegmentaciones}

Tabla 5. Distribución de hiposegmentaciones' por subcategorías y por decil.

\begin{tabular}{|c|c|c|c|c|c|c|c|c|c|c|c|c|}
\hline \multicolumn{2}{|c|}{$\begin{array}{l}\text { Deciles } \\
\text { Subcategoría }\end{array}$} & $1^{\circ}$ & $2^{\circ}$ & $3^{\circ}$ & $4^{\circ}$ & $5^{\circ}$ & $6^{\circ}$ & $7^{\circ}$ & $8^{\circ}$ & $9^{\circ}$ & $10^{\circ}$ & Total \\
\hline \multirow{2}{*}{$\begin{array}{l}\mathrm{HIPO} \\
\mathrm{PF}+\mathrm{PL}\end{array}$} & Frecuencia & & 12 & 11 & 10 & 16 & 18 & 28 & 42 & 41 & 38 & 216 \\
\hline & $\%$ & & 63,15 & 44 & 45,45 & 53,33 & 43,9 & 43,74 & 50 & 52,56 & 46,9 & 48,53 \\
\hline \multirow{2}{*}{$\begin{array}{l}\text { HIPO } \\
\mathrm{PF}+\mathrm{PF}\end{array}$} & Frecuenc & 1 & 6 & 9 & 5 & 9 & 20 & 27 & 19 & 22 & 15 & 133 \\
\hline & $\%$ & 100 & 31,57 & 36 & 22,72 & 30 & 48,78 & 42,18 & 22,61 & 28,2 & 18,51 & 29,88 \\
\hline \multirow{2}{*}{$\begin{array}{l}\mathrm{HIPO} \\
\mathrm{PL}+\mathrm{PL}\end{array}$} & Frecuenc & & 1 & 2 & 2 & 2 & 1 & 1 & 5 & 4 & 4 & 22 \\
\hline & $\%$ & & 5,26 & 8 & 9,09 & 6,66 & 2,43 & 1,56 & 5,95 & 5,12 & 4,93 & 4,94 \\
\hline \multirow{2}{*}{$\begin{array}{l}\mathrm{HIPO} \\
\mathrm{PL}+\mathrm{PF}\end{array}$} & Frecuenc & & & 2 & 3 & 1 & & & 4 & 5 & 6 & 21 \\
\hline & $\%$ & & & 8 & 13,63 & 3,33 & & & 4,76 & 6,41 & 7,4 & 4,71 \\
\hline \multirow{2}{*}{$\begin{array}{l}\text { HIPO } \\
\text { USC }\end{array}$} & Frecuencia & & & & & & & 1 & 7 & 3 & 11 & 22 \\
\hline & $\%$ & & & & & & & 1,56 & 8,33 & 3,84 & 13,58 & 4,94 \\
\hline \multirow{2}{*}{$\begin{array}{l}\text { HIPO } \\
\text { Otros }\end{array}$} & Frecuencia & & & 1 & 2 & 2 & 2 & 7 & 7 & 3 & 7 & 31 \\
\hline & $\%$ & & & 4 & 9,09 & 6,66 & 4,87 & 10,93 & 8,33 & 3,84 & 8,64 & 6,96 \\
\hline \multicolumn{2}{|c|}{ Total de frecuencia } & 1 & 19 & 25 & 22 & 30 & 41 & 64 & 84 & 78 & 81 & 445 \\
\hline \multicolumn{2}{|c|}{$\%$ del total de SNC } & 0,02 & 4,26 & 5,61 & 4,94 & 6,74 & 9,21 & 14,38 & 18,87 & 17,52 & 18,2 & 99,75 \\
\hline
\end{tabular}

Un primer aspecto que llama la atención es la variedad de tipos de respuestas (subcategorías de 'hiposegmentación') que se observa en casos con mayores y menores porcentajes de SNC en relación al total de palabras escritas. En los primeros deciles $\left(1^{\circ}\right.$ y $2^{\circ}$ decil en segundo grado y $2^{\circ}$ y $3^{\circ}$ decil en cuarto) las 'hiposegmentaciones' se asocian a un máximo de tres subcategorías de respuestas. Mientras que, en los últimos deciles, es decir en aquellos textos que tienen los porcentajes más altos de SNC, se observa también un mayor número de subcategorías. Podemos ver que en los últimos deciles $\left(8,9^{\circ}\right.$ y $10^{\circ}$ decil de ambos grados), las 'hiposegmentaciones' son de naturaleza más diversa, es decir, hay una combinación y organización mucho más variable: palabras léxicas que pueden anteceder o preceder a palabras funcionales; combinaciones de dos palabras léxicas o dos palabras funcionales y uniones de más de dos palabras, con distintas organizaciones. Un análisis más detallado del comportamiento de cada una de estas subcategorías por grado y decil servirá para ver con mayor profundidad lo que ocurrió con cada subcategoría de 'hiposegmentaciones'. Los resultados se presentarán en orden de frecuencia; excepto la subcategoría HIPO Otros que se presentará al final.

\section{HIPO PF+PL (Hiposegmentación de palabra funcional más palabra léxica)}

Las 'hiposegmentaciones' de palabras funcionales (artículos, pronombres, preposiciones, etc.) seguidas de palabras léxicas (sustantivos o verbos), por ejemplo: (1) seyama (se llama) o (2) aser (a ser), fueron las que alcanzaron los valores más altos, en términos absolutos y porcentuales: $48,53 \%$ en ambos grados. El análisis por grado reveló que el 53,38 \% y el 42,26\% de las 'hiposegmentaciones' en segundo y cuarto grado, respectivamente, corresponden a uniones de este tipo. 
Este tipo de cortes ha sido reportado en estudios realizados con niños de distintas lenguas, entre las que se encuentran el español, portugués, italiano y catalán (Díaz, 1992; Ferreiro \& Pontecorvo, 1996; Báez, 1999; Zamudio, 2004; Vaca, 2005; Bigas, 2006). Ahora bien, en relación a los criterios que orientarían este tipo de cortes, algunos de estos autores refieren el uso de patrones semánticos (Ferreiro \& Pontecorvo, 1996; Zamudio, 2004), que estarían motivados por el rechazo a dejar aisladas secuencias de letras que de forma independiente carecen de sentido (artículos, pronombres clíticos, preposiciones), enlazándolas a unidades de significación o palabras de sentido pleno (sustantivos, verbos, adjetivos, adverbios).

\section{HIPO PF+PF (Hiposegmentación de palabra funcional más palabra funcional)}

La unión de dos palabras funcionales es la segunda subcategoría más frecuente. En términos generales observamos que el $29,88 \%$ de las 'hiposegmentaciones' son de este tipo. Entre las estructuras más comunes se encuentran las uniones de preposiciones con artículos: (3) enla 'en la'; preposiciones con pronombres personales: (4) ami (a mî); preposiciones con pronombres demostrativos: (5) poreso (por eso); pronombres clíticos: (6) telo (te lo); conjunciones con pronombres clíticos: (7) ynos (y nos).

El análisis por deciles mostró que esta subcategoría de 'hiposegmentación' se encuentra en todos los grupos de segundo grado y desde el $3^{\circ}$ al $10^{\circ}$ decil en cuarto grado. En el caso de segundo grado se observó una disminución gradual de este tipo de 'hiposegmentaciones' a medida que aumenta la proporción de SNC, mientras que en los deciles de cuarto grado no se observa una tendencia clara.

Las dos subcategorías que hemos mencionado (HIPO PF+PL y HIPO PF+PF) concentran el 78,41\% del total de 'hiposegmentaciones' encontradas en ambos grados. Como veremos a continuación, las demás subcategorías representan porcentajes menores al 5\% del total de las 'hiposegmentaciones'.

\section{HIPO PL+PL (Hiposegmentación de palabras léxica más palabra léxica)}

Los análisis estadísticos dejaron ver que únicamente el 4,98\% del total de 'hiposegmentaciones' corresponden a uniones de dos palabras léxicas $(4,78 \%$ en segundo grado y 5,15\% en cuarto). Al analizar estos tipos de uniones como un solo grupo, no se observa un comportamiento claro: están presentes en varios deciles, tanto en textos con porcentajes bajos de SNC $\left(0,68 \% 2^{\circ}\right.$ decil de cuarto grado) como en grupos con altos porcentajes de $\mathrm{SNC}\left(8^{\circ}, 9^{\circ}\right.$ y $10^{\circ}$ de ambos grados). Sin embargo, sí constatamos diferencias al analizar más detalladamente las características de estos tipos de uniones en los primeros y últimos deciles. En los primeros grupos (entre el $2^{\circ}$ y $5^{\circ}$ decil) observamos secuencias que corresponden a unidades con coherencia sintáctica y semántica, principalmente a) uniones de dos sustantivos propios en la escritura de nombres compuestos: (8) Juancarlos (Juan Carlos); b) uniones de 
sustantivos comunes y propios: (9) villaHidalgo (Villa Hidalgo) y c) de sustantivos y adjetivos: (10) aigrecondicionario (aire acondicionado). No obstante, en deciles con mayores proporciones de SNC (entre el $7^{\circ}$ y el $10^{\circ}$ decil) se observan mayoritariamente uniones de verbos y sustantivos: (11) llamacarlos (llama Carlos).

\section{HIPO PL+PF (Hiposegmentación de palabra léxica más palabra funcional)}

Un tercer subgrupo de uniones con porcentajes cercanos al 5\% corresponde a la uniones de palabras léxicas precedidas por palabras gramaticales, generalmente uniones de verbos unidos a preposiciones: (12) Salimosa (Salimos a); sustantivos unidos a pronombres clíticos: (13) niñosse (niños se) y sustantivos unidos a conjunciones: (14) dulsesy (dulces y).

En el análisis por grado se pudo constatar que es el fenómeno que aparece en cuarto lugar en el caso de segundo grado $(5,97 \%$ ) y en el último lugar en cuarto grado $(3,09 \%)$. Asimismo, el análisis por decil evidenció que en segundo grado se observa en el $3^{\circ}, 4^{\circ}, 5^{\circ}, 8^{\circ}, 9^{\circ}$ y $10^{\circ}$ decil, mientras que solo se encuentra en los tres últimos deciles en cuarto grado, sin mostrar una tendencia clara en ninguno de los dos grados.

\section{HIPO USC (Hiposegmentación de dos o más palabras que integran una unidad sintáctica completa)}

El proceso de subcategorización de las 'hiposegmentaciones' se hizo utilizando como criterio principal el tipo de palabra involucrada (léxica o funcional), con independencia de la unidad mayor que podrían o no estar constituyendo. Sin embargo, observamos 'hiposegmentaciones' que se caracterizaban por afectar a más de dos palabras. En todos estos casos, la construcción de otras subcategorías siguiendo el mismo patrón (tipo de palabra) hubiera dado lugar a la construcción de subcategorías extensas con pocos datos, por ejemplo HIPO PL+PF+PF+PL para la escritura de (15) yegamosalaescuela (llegamos a la escuela) que hubieran dificultados los análisis estadísticos y resultado poco explicativas. Por esta razón, nos centramos en identificar qué tenían en común estas secuencias y encontramos dos grandes subgrupos: uno formado por secuencias que constituían unidades sintácticas completas y otro subgrupo en el que se encontraban unidades incompletas. El primero se etiquetó como HIPO USC y el segundo como HIPO Otros ('hiposegmentaciones' determinadas por otros criterios diferentes a la búsqueda de unidades sintácticas). En este apartado analizaremos las HIPO USC y en último lugar las HIPO Otros.

Las uniones de más de dos palabras que corresponden a una unidad sintáctica completa (HIPO USC) se presentan con un porcentaje de 4,94\% en relación al total de las 'hiposegmentaciones' (5,57\% en segundo y 4,12\% en cuarto grado). Se trata de uniones de más de dos palabras que coinciden con estructuras oracionales completas o alguna parte de éstas. Resultados similares fueron reportados por Vaca (2005) en 
relación a la segmentación de grandes constituyentes gramaticales de una oración (sujeto-predicado, sujeto-predicado-complemento), señalando la ocurrencia de estos tipos de fenómenos en proporciones similarmente bajas en casos de primero, segundo y cuarto grados y ausentes en casos de sexto grado ${ }^{5}$. En nuestros datos encontramos estructuras diferentes: oraciones completas (16) Comoesmiescuela (Cómo es mi escuela); frases preposicionales (17) que asemos enlaescuela (Qué hacemos en la escuela) y predicados completos de una oración (18) yo estoy contentaporconoserte (Yo estoy contenta por conocerte).

Aunque se trata de 22 escrituras de un total de 445, el modo en que se distribuyen por decil resulta muy interesante. Estos tipos de escrituras se observan en los últimos deciles (el $7^{\circ}, 8^{\circ}, 9^{\circ}$ y $10^{\circ}$ deciles en segundo grado y en $8^{\circ}, 9^{\circ}$ y $10^{\circ}$ en cuarto grado), con una tendencia a aumentar en término porcentuales a medida que aumenta la proporción de $\mathrm{SNC}$ en relación al total de palabras.

\section{HIPO Otros}

En esta subcategoría encontramos secuencias de dos tipos. Un primer tipo compuesto por escrituras continuas que combinan números y palabras, en contexto de escritura de fechas, grado, dirección, edad y otros: (19) 20deoctuvre '20 de Octubre'. $\mathrm{Y}$ un segundo grupo que corresponde a secuencias integradas por dos o más palabras que no constituyen una estructura sintáctica completa (20) otros nada máscrusanla calle (otros nada más cruzan la calle).

Que describamos en último lugar esta subcategoría de 'hiposegmentaciones', no significa que sea el grupo menos frecuente. De hecho, ocupa el tercer lugar en orden de frecuencia, con un porcentaje global de $6,96 \%$. El análisis por grado mostró un $5,97 \%$ en segundo grado y un $8,24 \%$ en cuarto. De modo similar, Vaca da cuenta de la categoría 'Inclasificable' que corresponde a casos en los que "no está gráficamente clara la fragmentación que realiza el niño" (Vaca, 2005: 61).

En nuestros datos, los análisis por decil mostraron que la subcategoría HIPO Otros no aparece en los primeros deciles, es decir, en el $1^{\circ}$ y $2^{\circ}$ decil en segundo grado y en el $1^{\circ}, 2^{\circ}$ y $3^{\circ}$ decil en cuarto grado. La proporción que se observa en el resto de los deciles, en ambos grados, no da cuenta de una tendencia claramente identificable. 


\subsubsection{Hipersegmentaciones}

Tabla 6. Distribución de 'hipersegmentaciones' por subcategorías y por decil.

\begin{tabular}{|c|c|c|c|c|c|c|c|c|c|c|c|c|}
\hline \multicolumn{2}{|c|}{$\begin{array}{ll}\text { Dubcategoría } & \text { Deciles }\end{array}$} & \multirow[t]{2}{*}{$1^{\circ}$} & \multirow{2}{*}{$\frac{2^{\circ}}{2}$} & \multirow{2}{*}{$\frac{3^{\mathbf{o}}}{2}$} & \multirow{2}{*}{$\frac{4^{\circ}}{1}$} & \multirow{2}{*}{$\frac{5^{\circ}}{12}$} & \multirow{2}{*}{$\frac{6^{\circ}}{10}$} & \multirow{2}{*}{$\frac{7^{\circ}}{14}$} & \multirow{2}{*}{$\begin{array}{l}8^{\mathbf{o}} \\
11\end{array}$} & \multirow{2}{*}{$\frac{9^{\circ}}{21}$} & \multirow{2}{*}{$\begin{array}{c}10^{\circ} \\
26\end{array}$} & \multirow{2}{*}{$\begin{array}{c}\text { Total } \\
99\end{array}$} \\
\hline \multirow{2}{*}{ HIPER PA } & Frecuencia & & & & & & & & & & & \\
\hline & $\%$ & & 100 & 28,57 & 20 & 80 & 43,47 & 46,66 & 35,48 & 53,84 & 30,23 & 41,59 \\
\hline \multirow{2}{*}{$\begin{array}{l}\text { HIPER } \\
\text { C SIL }\end{array}$} & Frecuencia & & & & 2 & 1 & 3 & 9 & 8 & 8 & 43 & 74 \\
\hline & $\%$ & & & & 40 & 6,66 & 13,04 & 30 & 25,8 & 20,51 & 50 & 31,09 \\
\hline \multirow{2}{*}{ HIPER DV } & Frecuencia & & & 2 & 1 & & 1 & 2 & 4 & 6 & 8 & 24 \\
\hline & $\%$ & & & 28,57 & 20 & & 4,34 & 6,66 & 12,9 & 15,38 & 9,3 & 10,08 \\
\hline \multirow{2}{*}{$\begin{array}{c}\text { HIPER } \\
\text { PLURAL }\end{array}$} & Frecuencia & & & & & & 2 & & 1 & & 1 & 4 \\
\hline & $\%$ & & & & & & 8,69 & & 3,22 & & 1,16 & 1,68 \\
\hline \multirow[b]{2}{*}{ HIPER AF } & Frecuencia & & & & & & & & & 2 & 1 & 3 \\
\hline & $\%$ & & & & & & & & & 5,12 & 1,16 & 1,26 \\
\hline \multirow{2}{*}{$\begin{array}{l}\text { HIPER SUF } \\
\text { MENTE }\end{array}$} & Frecuencia & & & & & & 1 & & 1 & & & 2 \\
\hline & $\%$ & & & & & & 4,34 & & 3,22 & & & 0,84 \\
\hline \multirow{2}{*}{$\begin{array}{l}\text { HIPER } \\
\text { Otros }\end{array}$} & Frecuencia & & & 3 & 1 & 2 & 6 & 5 & 6 & 2 & 7 & 32 \\
\hline & $\%$ & & & 42,85 & 20 & 13,33 & 26,08 & 16,66 & 19,35 & 5,12 & 8,13 & 13,44 \\
\hline \multicolumn{2}{|c|}{ total de frecuencia } & & 2 & 7 & 5 & 15 & 23 & 30 & 31 & 39 & 86 & 238 \\
\hline \multicolumn{2}{|c|}{$\%$ del total de SNC } & & 0,8 & 3,04 & 2,1 & 6,3 & 9,6 & 12,6 & 13,02 & 16,38 & 36,13 & 99,97 \\
\hline
\end{tabular}

De forma similar a lo que se observa en las 'hiposegmentaciones', en los primeros deciles (en el $3^{\circ}$ decil de segundo y entre el $2^{\circ}$ y el $5^{\circ}$ de cuarto) aparecen una o dos subcategoría de 'hipersegmentación' a diferencia de lo que muestran deciles con más altas proporciones de SNC. Tanto en segundo como en cuarto, en los últimos deciles $\left(9^{\circ}\right.$ y $10^{\circ}$ de segundo y $6^{\circ}, 8^{\circ}$ y $10^{\circ}$ de cuarto) se observa mayor diversidad de subcategorías, encontrándose hasta cinco diferentes.

\section{HIPER PA ('Hipersegmentaciones' de secuencias de letras que, en otros contextos, podrían funcionar como palabras independientes)}

Las 'hipersegmentaciones' más frecuentes (un porcentaje global de 41,59\%), tanto en segundo como en cuarto, corresponden a cortes en las palabras que dejan aisladas secuencias de letras que podrían constituir palabras gráficas convencionales en otros contextos. Se trata de un tipo de corte (configuraciones o secuencias gráficas autónomas) reportado por investigaciones precedentes (Díaz, 1992; Ferreiro \& Pontecorvo, 1996; Zamudio, 2004).

En nuestros datos, las secuencias gráficas más comunes son: es, como en (21) es cuela (escuela), a y de: (22) a prendemos (aprendemos) y (23) de sian (decían).

En un análisis por grado, pudimos observar que esta subcategoría aparece del $4^{\circ} \mathrm{al}$ $10^{\circ}$ decil, en segundo grado, con una tendencia a disminuir a medida que aumenta la proporción total de SNC y del $2^{\circ}$ al $10^{\circ}$ decil en cuarto grado (menos en el $4^{\circ}$ decil), en este caso, sin dar cuenta de una tendencia clara. 


\section{HIPER C SIL ('Hipersegmentaciones' de secuencias de letras que se corresponden con cortes silábicos)}

La segunda subcategoría más frecuente $(31,09 \%$ global) corresponde a recortes en los que parece incidir un criterio sonoro, que lleva a los niños a aislar unidades como son las sílabas, como (24) con Tre ras (Contreras). Los análisis estadísticos por grado muestran que el 28,77\% y el 34,34\% del total de las 'hipersegmentaciones', en segundo y cuarto grado respectivamente, corresponden a cortes de tipo silábico.

Los cortes silábicos, exhaustivos y no exhaustivos, han sido reportados por varios trabajos precedentes (Díaz, 1992; Ferreiro \& Pontecorvo, 1996; Zamudio, 2004; Vaca, 2005; Bigas, 2006). La mayoría de estos autores coinciden en señalar que un análisis fonológico, en este caso de segmentación silábica, parece ser el criterio que orienta estos tipos de cortes, principalmente, en momentos menos evolucionados del proceso de aprendizaje de la segmentación.

Estas dos subcategorías (HIPER PA e HIPER C SIL) constituyen juntas el 72,68\% del total de las 'hipersegmentaciones'. Encontramos otros tipos de 'hipersegmentaciones' que, aunque tienen los porcentajes más bajos, parecen estar guiados por análisis más finos de la estructura interna de las palabras. Nos referimos a las subcategorías: 'hipersegmentación de las desinencias verbales' (HIPER DV), 'hipersegmentación del morfema de plural' (HIPER PLURAL), 'hipersegmentación del afijo de una palabra' (HIPER AF) e 'hipersegmentación del sufijo -mente' en adverbios (HIPER SUF -MENTE). Se trata de cortes a los que también hace referencia Ferreiro y Pontecorvo (1996: 71): “también observamos en español (...) 'hipersegmentaciones' que pueden llevar a aislar (...) un morfema (nueva mente, des apareció, re lamiéndose)".

Bajo la categoría HIPER DV ('hipersegmentación' de las desinencias verbales) agrupamos las fragmentaciones de verbos que dan lugar a la separación de series de letras que coinciden con alguna de sus desinencias. La mayoría corresponde a la marca de la primera persona del plural, en la escritura de acciones habituales que realizan los niños en la escuela: (25) llega mos (llegamos).

En el análisis por grado se observa que el 12,94\% de segundo y el 6,06\% de cuarto, corresponden a segmentaciones este tipo. El análisis por decil mostró que aparecen en la mayor parte de los deciles de segundo grado (menos en el $1^{\circ}, 2^{\circ}$ y $5^{\circ}$ ) y en los últimos deciles de cuarto grado $\left(7^{\circ}, 8^{\circ}\right.$ y $\left.10^{\circ}\right)$, sin mostrar una tendencia clara.

Otro tipo de segmentaciones con muy baja frecuencia de aparición corresponde a cortes del morfema de número (HIPER PLURAL) como (26) hermano s (hermanos) De un total de cuatro secuencias, tres fenómenos se observan en casos de cuarto grado $\left(6^{\circ}, 8^{\circ}\right.$ y $10^{\circ}$ decil $)$ y uno en segundo grado $\left(6^{\circ}\right.$ decil $)$. 
También encontramos tres 'segmentación del afijo de una palabra' (HIPER AF), como (27) re cortamos (recortamos) que, aunque representan el 2\% (3 de 236) del total de 'hipersegmentaciones', resulta interesante analizar por separado. Se trata de tres cortes que realizan dos casos de segundo grado y que impresionan como intentos por encontrar prefijos en la palabra $\left(9^{\circ}\right.$ y $10^{\circ}$ decil). Esto podría indicar que, si bien muy bajas proporciones, las segmentaciones guiadas por cortes morfológicos se presentan en distintos momentos de la evolución del conocimiento y no solo en niveles más avanzados.

Algo similar ocurre con las HIPER SUF -MENTE (morfema 'mente' en adverbios) como (28) procima mente (próximamente), de las cuáles encontramos dos secuencias. A diferencia de lo que ocurre con los afijos, estos tipos de segmentaciones ocurrieron solo en casos de cuarto grado, presentes en el $6^{\circ}$ y $8^{\circ}$ decil.

Respecto al criterio morfológico, Ferreiro y Pontecorvo (1996) señalan que la poca frecuencia de estos tipos de cortes se explica porque los niños no usarían tempranamente este patrón de separación. Asimismo, Rayas (2009) apoyándose en lo indicado por BryanT y Nunes (2006), señala que la mayor experiencia de los sujetos con la lengua escrita es lo que permite que se hagan sensibles a encontrar unidades morfológicas. Desde este último supuesto, podríamos esperar que cortes de tipo morfológico fueran más frecuentes en casos de cuarto grado. Nuestros datos no resultan concluyentes al respecto. HIPER PLURAL se encuentra en tres deciles de cuarto grado y en uno de segundo; HIPER AF se observa solo en casos de segundo; HIPER DV aparece en la mayor parte de los deciles de segundo grado y en los últimos tres deciles de cuarto; por su parte HIPER SUF -MENTE corresponde solo a dos casos de cuarto, ubicados en el $6^{\circ}$ y $8^{\circ}$ decil.

Finalmente, encontramos 'hipersegmentaciones' que no se corresponden con ninguna de las subcategorías anteriores. Agrupamos bajo la categoría HIPER Otros separaciones de una sola grafía, que no coinciden con letras o secuencias gráficas que, en otros contextos, podrían funcionar como palabras independientes, por ejemplo: (29) pisarro $\mathbf{n}$ (pizarrón) y cortes que ocurren en medio de palabras y que no coinciden con las fronteras silábicas, como son: (30) vivil oteca (biblioteca).

Si bien resulta complejo identificar el o los criterios que parecen guiar estos tipos de cortes, los análisis cuantitativos indican que en segundo grado se concentran en los últimos deciles, mientras que en cuarto se observan en casi todos los deciles (menos en el $1^{\circ}$ y $5^{\circ}$ decil), sin mostrar en ninguno de los grados una tendencia clara a medida que aumenta la proporción de SNC por el total de palabras. 


\subsubsection{Hipo-hipersegmentaciones}

El 5\% del total de SNC corresponden a escrituras en las que dos o más palabras se ven afectadas simultáneamente por fenómenos de 'hiposegmentación' e 'hipersegmentación'. Muchas de estas secuencias unen palabras funcionales y palabras léxicas, segmentado a la vez en dos partes las unidades de sentido pleno: (31) miPa Pá (mi papá). Otras, unen dos palabras léxicas, fragmentando al mismo tiempo una de estas unidades: (32) esgran de (es grande). También, encontramos escrituras semejantes a las del tipo USC (dos o más palabras que integran una unidad sintáctica completa), que a la vez segmentan indebidamente uno de sus elementos: (33) yega nosalaescuela (llegamos a la escuela).

Aunque en términos absolutos son pocos fenómenos en segundo y cuarto grado (37 de un total de 720), el modo en que se distribuyen por decil resulta muy ilustrativo. Escrituras que combinan en una sola secuencia 'hiposegmentaciones' e 'hipersegmentaciones' se observan principalmente en los últimos deciles, tanto en segundo y cuarto grado. Hay que señalar que estos 37 casos no se tomaron en consideración en los análisis anteriores.

\subsection{Criterios de segmentación}

Respecto de los criterios que estarían detrás de las decisiones de los niños, resulta complejo definir qué patrón o patrones específicos explican las distintas subcategorías de segmentaciones que identificamos en los textos analizados. Sin embargo, podemos formular algunas hipótesis que tienen que ser corroboradas con otros tipos de análisis y datos. Según señala Zamudio (2004), factores vinculados a la oralidad (criterios entonacionales o acentuales y de corte silábico), parecen estar detrás de 'hiposegmentaciones' de más de dos palabras que corresponden a unidades sintácticas completas y de cortes guiados por criterios silábicos. Resultó interesante observar en nuestros datos que ambos fenómenos aparecen sistemáticamente en los grupos menos avanzados y no se observan en los grupos con proporciones más bajas de SNC. Lo que propone esta autora y nuestros análisis cualitativos permiten pensar que en niveles iniciales del aprendizaje de la segmentación, el criterio fonológico coexiste con otros criterios de tipo gramatical y gráfico, que el uso simultáneo de esta diversidad de patrones explicaría la abundancia de SNC en estos textos y que, a medida se avanza en el conocimiento de la escritura, los criterios fonológicos se irían descartando a favor de criterios gráficos y gramaticales.

Del mismo modo, qué criterios están detrás de las segmentaciones más frecuentes (uniones palabra gramatical más palabra léxica y a las secuencias palabra autónoma) es tema de debate entre los autores revisados (Ferreiro \& Pontecorvo, 1996; Zamudio, 2004; Vaca, 2005; Bigas, 2006). En nuestros datos, estos tipos de cortes se observan en grupos con altas y bajas proporciones de SNC, la diferencia está en que en los grupos más avanzados, las pocas SNC que realizan corresponden generalmente a estos 
tipos de cortes, mientras que en casos con numerosas SNC, estos cortes más habituales coexisten con otros que a primera vista parecen más insólitos (palabras léxicas que preceden a palabras funcionales, aislamiento de unidades sintácticas completas, etc.) y por tanto más complejos de explicar.

Nos resulta muy interesante lo que observamos respecto de las uniones palabra gramatical más palabra léxica. Por una parte, se trata de unidades muy sólidas en las que convergen varios criterios: podrían quedar unidas porque son una unidad sintáctica y semántica en sí mismas y/o porque aisladamente las palabras gramaticales carecen de significado o porque carecen de carga tónica o porque son secuencias gráficas de pocas letras. Esta diversidad de razones podría estar detrás de la frecuencia y transversalidad en su aparición. La persistencia y frecuencia de estos tipos de uniones demuestra que se trata de unidades que exigen mayor conocimiento y ejercicio intelectual de combinatorias para llegar a comprender que son unidades gráficamente independientes.

En cuanto al criterio morfológico, coincidimos con lo que proponen Ferreiro y Pontecorvo (1996) y Zamudio (2004): son cortes poco frecuentes y esto se podría explicar porque los niños no usarían tempranamente este patrón de separación. Sin embargo, nuestros datos no son suficientes para afirmar que a más experiencia con la escritura, más posibilidades de aislar unidades morfológicas como lo sostiene Rayas, (2009). Encontramos separaciones que coinciden con cortes morfológicos en sujetos de segundo y cuarto y grado y en casos con altas y bajas proporciones de SNC.

\section{CONCLUSIONES}

Respecto de los tipos de SNC, encontramos distintos resultados según el criterio de agrupación de los textos. Los análisis por grado coinciden con lo señalado por otras investigaciones de referencia: las 'hiposegmentaciones' son más frecuentes que las 'hipersegmentaciones' y que la mezcla de ambas. Al agrupar los textos según proporción de SNC (organización en deciles) observamos otras formas de comportamiento. En segundo grado, los textos con menos problemas de segmentación tienen exclusivamente 'hiposegmentaciones', mientras que las 'hipersegmentaciones' aumentan de forma gradual, llegando a tener, en los casos menos avanzados, un peso semejante al de las 'hiposegmentaciones'. En textos de cuarto grado, sin embargo, las 'hiposegmentaciones' son significativamente más frecuentes en casi todos los deciles, excepto en los extremos, es decir, los que tienen la proporción más baja y más alta de SNC. Las 'hipo-hipersegmentaciones' siguen un patrón muy claro: aparecen, tanto en segundo como en cuarto grado, solo en los textos con mayor proporción de problemas de segmentación y aumentan a medida esta misma proporción aumenta. 
Con respecto a la variedad de subcategorías encontradas en cada decil podemos señalar que en casos con pocas SNC, las 'hiposegmentaciones' e 'hipersegmentaciones' se asocian a dos o tres subcategorías de respuestas. Las segmentaciones no convencionales (SNC) más frecuentemente observadas en nuestros análisis y que también han sido descritas en otros estudios son las uniones de palabra funcional más palabra léxica (HIPO PF+PL); uniones de dos palabras funcionales (HIPO PF+PF); separaciones de letras que coinciden con la escritura de otras palabras (HIPER PA ) y de secuencias que se ubican en cortes silábicos de la palabra (HIPER C SIL). En el caso de los niños con altas proporciones de SNC, las subcategorías mencionadas aparecen junto a otros tipos de uniones y separaciones menos habituales como las de palabra léxica más palabra léxica (HIPO PL+PL), palabra léxica seguida de palabra funcional (HIPO PL+PF); o cortes que aíslan un morfema de la palabra (HIPER PLURAL, AF o -MENTE), entre otros. Estos resultados permiten afirmar que en niveles iniciales del conocimiento los niños exploran diversos criterios y posibilidades de separación de las palabras, variedad de criterios que irán abandonando para quedarse con solo unos pocos a medida aumenta el conocimiento de la escritura.

Finalmente, sostenemos que los datos aquí presentados confirman que las SNC que se observan en la escritura infantil responden a un ejercicio racional que realizan los niños por comprender la naturaleza de las unidades que la escritura separa. El uso de espacios en blanco delimitando unidades, diferentes a las palabras, como pueden ser las sílabas, los morfemas o unidades gráficas que podrían constituir 'palabras' en el español, así lo demuestran. En futuras investigaciones, resulta interesante complementar con otros tipos de estrategias, como son las entrevistas, y ampliar el análisis considerando las segmentaciones convencionales y las unidades superiores en las que aparecen segmentaciones no convencionales. 


\section{REFERENCIAS BIBLIOGRÁFICAS}

Alegría, M. (2014). Evolución en la segmentación de la escritura. El uso de los espacios en blanco en textos de niños que inician su alfabetización. Tesis de magíster, Universidad Nacional de La Plata, La Plata, Argentina.

Báez, M. (1999). La problemática de segmentar el texto escrito en palabras: Una indagación psicolingüística. En M. Báez \& V. Cárdenas (Eds.), Segmentación y escritura. Dos estudios sobre adquisición (pp. 11-69). Rosario: Homo Sapiens.

Béguelin, M. J. (2002). Unidades de lengua y unidades de escritura. Evolución y modalidades de la segmentación gráfica. En E. Ferreiro (Ed.), Relaciones de (in) dependencia entre la oralidady la escritura (pp. 31-52). Barcelona: Gedisa.

Bigas, M. (2006). La segmentació del text escrit en infants de primer curs de primària. Estudi evolutiu. Tesis doctoral, Universitat Autònoma de Barcelona, Barcelona, España.

Blanche-Benveniste, C. (1998). Estudios lingüisticos sobre la relación entre oralidad y escritura. Barcelona: Gedisa.

Bryant, P. \& Nunes, T. (2006). Improving literacy by teaching morphemes. Londres: Routledge.

Díaz, C. (1992). La segmentación en la escritura. El caso de los cliticos en el español. Tesis de maestría, Centro de Investigación y de Estudios Avanzados del Instituto Politécnico Nacional, D.F, México.

Ferreiro, E. (2012). Comprensión del sistema alfabético de escritura. En M. Carretero \& J. A. Castorina (Comps.), Desarrollo cognitivo y educación II. Procesos del conocimiento y contenidos específicos (pp. 290 -300). Buenos Aires: Paidós.

Ferreiro, E. \& Teberosky, A. (1979). Los sistemas de escritura en el desarrollo del niño. México: Siglo XXI Editores.

Ferreiro, E. \& Vernon, S. (1992). La distinción palabra/nombre en niños de 4 y 5 años. Infancia y Aprendizaje, 58, 15-28.

Ferreiro, E. \& Pontecorvo, C. (1996). Los límites entre las palabras. En E. Ferreiro, C. Pontecorvo, N. Ribeiro \& I. García (Eds.), Caperucita Roja aprende a escribir (pp. 45-71). Barcelona: Gedisa.

Fruyt, M. \& Reichler- Béguelin, J. M. (1990). La notion de 'mot' en latin et dans d'autres langues indo-européennes anciennes. Modèles Linguistiques, XII (1), 21 46. 
González, E. (2009). Variables extralingüísticas en la segmentación anómala por niños de Maracaibo. Opción, 25(60), 81-96.

OREALC/UNESCO (2010). SERCE. Segundo estudio regional comparativo. Escritura. Un estudio de las habilidades de los estudiantes de América Latina y el Caribe [en línea]. Disponible en: http://unesdoc.unesco.org/images/0019/001919/191925s.pdf

Pérez, (2006). La travesía de la escritura. De la cultura oral a la cultura escrita. México: Santillana.

Querejeta, M., Piacente, T., Guerrero, H. \& Alva, A. (2013). La separación entre palabras en la escritura de niños que inician la escolaridad primaria. Revista Interdisciplinaria, 30(1), 45-64.

Rayas, L. (2009). Evolución y persistencia de las segmentaciones gráficas no convencionales. Tesis de magíster, Universidad Autónoma de Querétaro, Querétaro, México.

Reyes, M. (2014). Razonamiento de niños de 7 y 8 años al hipo e hipersegmentar las palabras cuando reescriben un texto narrativo. Tesis de magíster, Instituto de Psicología, Universidad del Valle, Cali, Colombia.

Saenger, P. (1997). Space between words, the origins of silent reading. California: Stanford University Press.

Sotomayor, C., Molina, D., Bedwell, P. \& Hernández, C. (2013). Caracterización de problemas ortográficos recurrentes en alumnos de escuelas municipales chilenas de $3^{\circ}, 5^{\circ}$ y $7^{\circ}$ básico. Revista Signos. Estudios de Lingüistica, 46(81), 105 131.

Vaca, J. (2005). La segmentación de oraciones escritas por niños de 7 a 12 años. Puertas a la lectura, 18, 58-70.

Zamudio, C. (2004). Las ideas infantiles sobre la separación de las palabras. En A. Pellicer \& S. Vernon (Eds.), Enseñar la lengua escrita en la escuela (pp.71-95). México: SM Editores.

Zamudio, C. (2010). Las consecuencias de la escritura alfabética en la teoría lingüistica. México: El Colegio de México.

Zamudio, C. \& Díaz, C. (2011). Desarrollo de una herramienta informática para el análisis de textos infantiles. Ponencia presentada en el XVI Congreso Internacional de la ALFAL, Universidad de Alcalá, Alcalá de Henares, España. 


\section{NOTAS}

${ }^{1}$ Los resultados de este trabajo son parte de una tesis para optar al grado de Magíster en Escritura y Alfabetización en la Universidad Nacional de La Plata, Argentina.

${ }^{2}$ El Corpus Electrónico para el Estudio de la Lengua Escrita (CEELE) se puede consultar en: http://www.corpus.unam.mx:8080/ceele/

${ }^{3}$ En estadística el concepto decil se refiere a cada uno de los 9 valores que dividen un grupo de datos clasificados con una relación de orden en diez partes iguales.

4 Para ver los análisis independientes de cada grado se sugiere consultar: http://hdl.handle.net/10915/35145

${ }^{5}$ En su estudio de escritura de oraciones al dictado, Vaca (2006) da cuenta de 6 tipos de respuestas: 1) ninguna fragmentación $(0,9 \%) ; 2)$ en grandes constituyentes $(3,8 \%) ; 3)$ silábica $(4,7 \%) ; 4)$ inclasificable $(6,4 \%) ; 5)$ de constituyentes $(22,4 \%)$ y 6$)$ convencional $(61,8 \%)$. 